\title{
Perirhinal Cortex Removal Dissociates Two Memory Systems in Matching-to-Sample Performance in Rhesus Monkeys
}

\author{
Hsiao-Wei Tu, ${ }^{1}$ Robert R. Hampton, ${ }^{1}$ and Elisabeth A. Murray ${ }^{2}$ \\ ${ }^{1}$ Department of Psychology and Yerkes National Primate Research Center, Emory University, Atlanta, Georgia 30322, and ${ }^{2}$ Section on the Neurobiology of \\ Learning and Memory, Laboratory of Neuropsychology, National Institute of Mental Health, National Institutes of Health, Bethesda, Maryland 20892
}

\begin{abstract}
Dissociations of memory systems are typically made using independent cognitive tests. For example, in monkeys habits have been inferred from performance in object discrimination tests, while non-matching-to-sample tests are thought to measure familiarity resulting from single exposures. Such tests cannot measure individual memory processes accurately when more than one memory process contributes to performance. In process dissociation procedures (PDPs), two memory processes cooperate and compete in the performance of a single cognitive task, allowing quantitative estimates of the contributions of each process. We used PDP to measure the contributions of habits and one-trial memory to visual matching-to-sample performance. Sets of test images were shown only once in each daily testing session but were repeated day after day. To produce habits, high-frequency images were correct more frequently than other images across days. Habits were manifest in the extent to which choices in the test phase of matching-to-sample trials were made to the high-frequency images, regardless of which image had been presented as the sample. One-trial memory was measured by the extent to which choices at test were made to the image that had appeared as the sample on that trial, regardless of habit. Perirhinal cortex removal reduced the contribution of one-trial memory to matching performance, but left both habits and the ability to discriminate images intact. PDP can be applied in monkeys in a way that parallels its use in humans, providing a new tool for investigating the neurobiology of memory in nonhuman animals and for comparing memory across species.
\end{abstract}

\section{Introduction}

At least two memory systems can be characterized based on processing modes: one supports slow incremental learning of habits and skills; the other supports rapid one-trial learning of items and associations (Mishkin et al., 1984; Bachevalier, 1990; Henke, 2010). In humans, the distinction between these two systems often hinges on verbal reports of private experience. The habit system is automatic and not accessible to subjective monitoring or commentary, while the one-trial system is cognitively controlled and accessible to subjective monitoring (Hay and Jacoby, 1996). Both systems may operate in many tasks (Jacoby, 1991; Hay and Jacoby, 1996), which prevents equating performance in a specific task with the operation of a particular memory system.

In monkeys, delayed non-matching-to-sample has been used to measure one-trial memory, and object discrimination learning has been used to measure habit formation (Mishkin et al., 1984). It is unlikely that either test measures just one memory process.

Received May 7, 2011; revised Aug. 24, 2011; accepted Sept. 21, 2011.

Author contributions: R.R.H. and E.A.M. designed research; R.R.H. performed research; H.-W.T. analyzed data; H.-W.T. wrote the paper.

This work was supported by the Intramural Research Program of the National Institute of Mental Health (NIMH). Additional support was provided by NIMH Grant R01MH082819, National Science Foundation Grant 0745573, and Yerkes Center Base Grant RR-00165, which was awarded by the Animal Resources Program of the National Institutes of Health. We thank Sparky Zivin for assistance with testing animals and animal care; Rick Duntz for assistance in surgery; and Jeanette Black and Renee Hill of the NIH In Vivo Nuclear Magnetic Resonance Research Center for technical assistance.

Correspondence should be addressed to Hsiao-Wei Tu, Yerkes National Primate Research Center, Emory University, 954 Gatewood Road, Atlanta, GA 30329. E-mail: hsiaowei.tu@emory.edu.

DOI:10.1523/JNEUROSCI.2338-11.2011

Copyright $\odot 2011$ the authors $\quad 0270-6474 / 11 / 3116336-08 \$ 15.00 / 0$
For example, in learning set (Harlow, 1949) and serial reversal learning (Schrier, 1966), gradual learning is initially observed, but after extensive experience monkeys learn new stimulus-reward associations in a single trial. The slow initial learning, followed by rapid terminal learning, suggests a shift from predominantly habitbased learning to one-trial memory, even though the task, discrimination learning, remains the same.

Process dissociation procedures (PDPs) offer one solution to the problems inherent in equating tests with specific cognitive processes (Jacoby, 1991; Kelley and Jacoby, 1998, 2000). Using PDP, one assumes that habit and one-trial memory are active simultaneously and independently. PDP allows quantification of the contributions of these two processes in a single task. Experiments in humans demonstrate that measures of habit and onetrial memory from PDP correspond with those derived from traditional tasks argued to be "process-pure" (Jacoby et al., 1993; Toth et al., 1994; Reingold and Goshen-Gottstein, 1996). Use of PDP with nonhumans has not been reported.

We used PDP in rhesus monkeys (Macaca mulatta) to assess the contributions of perirhinal cortex (PRh) to memory. Specifically, we tested for a dissociation of habit and one-trial memory by comparing the performance of monkeys with and without perirhinal cortex in a modified matching-to-sample test. By using identical visual stimuli, presented in the identical task context, in tests with the same subjects, we controlled for factors other than memory type that might cause differences in performance across separate memory tests. These factors include the type and the discriminability of the stimuli, size of the image set, frequency of image repetition, motivation of the subjects, and time after sur- 
gery. If monkeys with PRh lesions exhibit deficits in both processes, this would be consistent with a role for PRh in both habit and one-trial memory. By contrast, a selective deficit in one-trial memory would indicate that $\mathrm{PRh}$ plays a role in memory that is independent of the contribution it makes to habits. The latter finding would leave us agnostic about some perceptual functions of the PRh, because perceptual functions were not taxed, but it would indicate that memory impairments can occur independent of perceptual deficits.

\section{Materials and Methods}

\section{Subjects}

Eight male and two female adult rhesus monkeys were used. The animals weighed $6.2-12.2 \mathrm{~kg}$ at the beginning of testing, were caged individually, and were fed daily an amount of biscuits and fruit adjusted for each animal to ensure sufficient motivation and nutrition. Water was always available in the home cage. Six animals had been operated on to remove the perirhinal cortex bilaterally (group PRh) (Hampton and Murray, 2002); the remaining four animals served as unoperated controls (group Con). Each group included one of the two females. The monkeys had prior experience in automated cognitive testing. The study was conducted under a protocol approved by the Animal Care and Use Committee of the National Institute of Mental Health and in accordance with the Guide for the Care and Use of Laboratory Animals of the National Institutes of Health.

\section{Surgery}

The surgical procedures used to remove the perirhinal cortex are described in detail in Hampton and Murray (2002). Briefly, under isoflurane anesthesia, a large bone flap was turned over each hemisphere, and the dura reflected in two locations to permit anterior and posterior access to perirhinal cortex. The tissue on the lateral bank of the rhinal sulcus was removed, together with $2-3 \mathrm{~mm}$ of cortex just lateral to the sulcus, by subpial aspiration through a small gauge sucker. The opening was then closed in anatomical layers. Dexamethasone sodium phosphate $(.5 \mathrm{mg} /$ $\mathrm{kg}$ ) and cefazolin were administered $1 \mathrm{~d}$ before surgery and for 1 week after surgery to reduce inflammation and to prevent infection, respectively. For 3-5 d after surgery, the monkeys received Banamine (1.0 $\mathrm{mg} / \mathrm{kg}$, b.i.d.) and acetaminophen (10 mg/kg, b.i.d.) for analgesia.

Assessment of lesions using MRI

Details on the use of in vivo MRI to assess the extent of the perirhinal cortex removals in these monkeys are described by Hampton and Murray (2002). Other work has demonstrated good agreement between in vivo MRI and postmortem histology in the assessment of temporal lobe lesions (Bachevalier and Mishkin, 1994; Bachevalier et al., 1999; Liu et al., 2000; Málková et al., 2001). The lesion in each monkey was evaluated on coronal MR images and plotted at $1 \mathrm{~mm}$ intervals onto drawings of coronal sections of a standard rhesus monkey brain. The areas of perirhinal cortex, area TE, area TG, and entorhinal cortex were determined in each standard section using a digitizer and computer software $\mathrm{NIH}$ Image). Lesion volumes were then determined for each monkey, and the size of the lesion expressed as a proportion of the standard.

\section{Apparatus}

Monkeys were trained using an automated apparatus controlled by an IBM compatible computer. During test sessions, monkeys were seated in primate chairs inside ventilated sound-attenuating chambers and positioned in front of 14 inch color video monitors fitted with touch-sensitive screens. To the left and right of the monitors were two food cups for the automated delivery of peanuts and $190 \mathrm{mg}$ primate pellets, respectively. The test chambers were illuminated by $15 \mathrm{~W}$ light bulbs. Visual stimuli were 288 color images (Corel Mega Gallery clip art), 160 pixels high $\times$ 200 pixels wide $(43 \times 53 \mathrm{~mm})$.

\section{Visual matching-to-sample}

Test images were randomly assigned to "image quads" such that fixed sets of four images were always presented together over the course of the experiments. A trial started with a study phase in which one of the four images in a given image quad was selected for use as the sample and was presented centrally on the computer monitor. Monkeys touched the sample twice (FR2), after which it disappeared. After a delay during which the screen was black, the test phase started when all four images in that image quad appeared, randomly assigned to the four corners of the monitor. Monkeys were rewarded for touching (FR2) the image seen during the study phase of that trial. To retard satiation, half of correct responses were rewarded with a peanut, and the other half with a primate pellet, on a random schedule. Following incorrect responses, the screen turned purple for a $5 \mathrm{~s}$ timeout period. When the correction procedure was in effect, the correct image then appeared by itself in the corner where it had just appeared during the test phase. Touching (FR2) this image resulted in reward as on correct trials. An intertrial interval of $10 \mathrm{~s} \mathrm{sepa-}$ rated trials.

\section{Behavioral procedure}

Experiment 1: matching-to-sample. All monkeys had been familiarized with the apparatus and the visual matching-to-sample task before this study. Immediately before beginning the current experiment, monkeys were retrained in visual matching-to-sample until performance reached $90 \%$ correct for three consecutive sessions. Selection of sample images from the image quads was random during this phase; each image was equally likely to appear as the sample. The experiment proper followed, consisting of 15 48-trial sessions with six randomly intermixed programmed delays $(0,2,4,8,16$, and $32 \mathrm{~s}$; each used eight times in each session) with a set of 56 image quads (224 images total). The correction procedure was not used in this experiment.

Experiment 2: $70 \%$ bias. A new set of 72 image quads was used (288 images), each of which only appeared once in each session but were reused day after day. The order of the 72 image quads was randomly determined for each session. Initially, one image was randomly selected from each image quad to be the sample on each trial. Monkeys completed one 72-trial session per day, 5-7 d a week until sessions were completed in $<40 \mathrm{~min}$ for 5 consecutive days. A single programmed delay of $0 \mathrm{~s}$ was used. The correction procedure was in effect for incorrect trials.

Subsequent trials proceeded as before, except that the selection of which image from each image quad would serve as the sample was no longer random. Instead, one of the four images in each image quad was designated the high-frequency image, and it served as the sample with a probability of 0.7 . Each of the other three images in each quad served as the sample with a probability of 0.1 (Fig. 1). Thus, while the correct response on a given trial was always to choose the image seen during study on that trial, one image in each image quad was correct $70 \%$ of the time across sessions. In other words, monkeys could achieve $70 \%$ correct responses, but no better, by always choosing the high-frequency images at test, whereas $100 \%$ accuracy could be achieved only by remembering the sample image seen on each trial. The correction procedure ensured that all trials ended with a rewarded response to the correct image for that trial. Monkeys completed 25 sessions under these conditions.

In the final phase of testing, 24 trials were randomly selected in each session as probes and the other 48 trials were exactly the same as before. There were three types of probes, eight trials of each type. Congruent probe trials began with presentation of a high-frequency image as the sample, followed by a $60 \mathrm{~s}$ delay, during which the screen was black. At test, monkeys were rewarded for selecting the image that had appeared as the sample on that trial. Thus, both one-trial memory and habit correspond to the same response on congruent probe trials; the two memory systems acted in cooperation. Incongruent probe trials were identical to congruent probes, except that a low-frequency image served as the sample. Again monkeys were rewarded for choosing the image that had appeared as the sample on that trial. Because the sample on these trials was not the high-frequency image, habit and one-trial memory corresponded to different choices at test; the two memory systems acted in opposition. On empty probe trials, no sample image was presented at the beginning of the trial. Following the end of the intertrial interval, the image quad for that trial was presented. Thus, habit acted in the absence of any opposition or cooperation from one-trial memory from the study phase of the trial. Monkeys were rewarded on empty probe trials regardless of choice. The correction procedure was not used with any probe trials. Fifteen 
probe sessions were conducted, yielding 120 trials of each probe type for analysis.

Experiment 3: $100 \%$ bias. This experiment was the same as Experiment 2, and used the same image quads, but now the high-frequency images served as the sample on $100 \%$ of trials. Monkeys completed 15 sessions under these conditions and were then given 15 probe sessions as in Experiment 2.

Data analysis: process dissociation. The probability that the high-frequency images were chosen on congruent probes (correct responses) and on incongruent probes (errors) was determined. The contributions of habit and one-trial memory to these values can be expressed as shown below, where $p(h f)$ is the probability of selecting the high-frequency images (Jacoby, 1991; Hay and Jacoby, 1996). Note that the $p(h f)$ is not equivalent to accuracy. It represents the probability that monkeys chose the high-frequency image, regardless of which image was correct: congruent: $p(h f)=$ one-trial memory + habit (1 - one-trial memory); incongruent: $p(h f)=$ habit $(1-$ one-trial memory).

Thus, the probability of selecting the highfrequency images on congruent trials, when it is the correct choice, reflects the probability of one-trial memory plus the conjoint probability of habit and the failure of one-trial memory. Monkeys select the high-frequency image either because they remember it from the study phase of the trial, or because they have a habit to do so even though they forgot seeing it as the sample. In contrast, monkeys select the highfrequency image on incongruent trials, an error, only when they have forgotten which image appeared as the sample, and they have a habit to select the high-frequency image. Note that the equations for these two circumstances share the term "habit (1 - one-trial memory)," which reflects the conjoint probability of habit on trials when one-trial memory failed. Therefore, the value $p(h f)$ for incongruent probes, which was determined directly from the behavioral data, can be substituted into the equation for congruent probes, permitting a solution for the value of one-trial memory. The value for one-trial memory can then be entered into the formula for incongruent probes to solve for the value of habit.

Empty probes provide a manipulation check. Because no sample was presented on empty probes, the probability of choosing the high-frequency image on these trials should be largely a pure measure of habit; there is no one-trial memory from the current trial to contribute to performance (empty: $p=$ habit).

Statistics. Proportional data were arcsine transformed before analysis to better conform to the assumption of normality (Kirk, 1982). Most results were analyzed first using within-subjects ANOVAs. Two-tailed post hoc $t$ tests were then used to further specify the source of significant differences found by ANOVA.

\section{Results}

\section{Lesion assessment}

The intended lesion, a representative MRI scan, and a plot of the lesion in Case PRh-E are shown in Figure 2. The percentage damage for each of the six animals in Group PRh is reported in Table 1. For further details, see Hampton and Murray (2002).

In all cases except PRh-C, discussed below, damage to perirhinal cortex approximated the intended lesion. Five of six animals had minor unintended damage to entorhinal cortex, ranging from near 0 to $10 \%$ (Table 1 ). Area TG was damaged in every
Sample
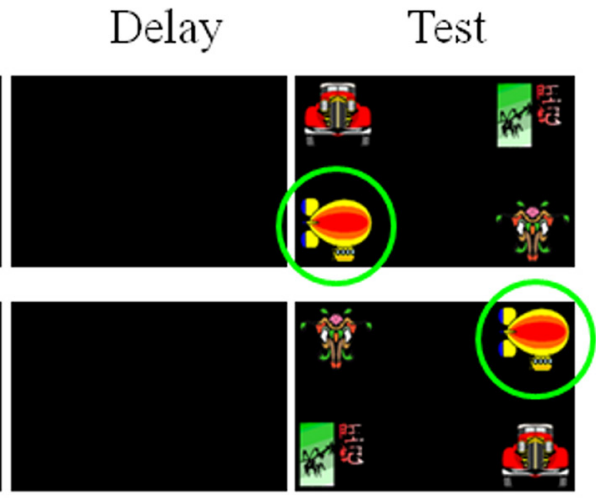

.

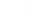
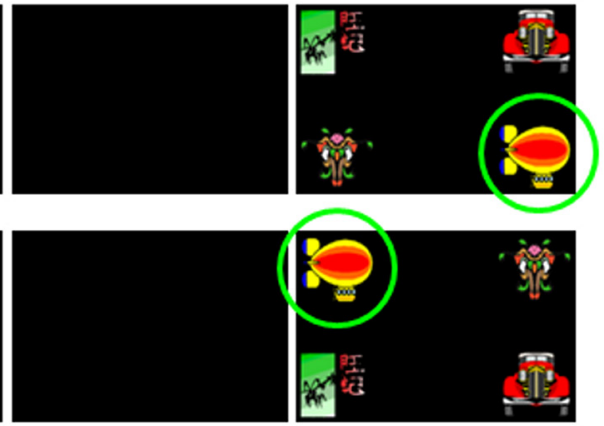

Figure 1. Each image quad appeared once in each daily testing session. Because one of the four images in each image quad was the sample more frequently than the others, there is opportunity for habits to select these high-frequency images to develop shows a high-frequency image appearing as the sample on every single trial, as in Experiment 3. In Experiment 2, the three images in each image quad that were not the high-frequency image each appeared as the sample with a probability of 0.1 .

monkey, $13.1 \%$ on average. Each monkey sustained a small amount of damage to the laterally adjacent area TE, but for five of the six monkeys, this damage did not exceed $4 \%$ of the volume of TE, averaged across the two hemispheres. Case PRh-C was observed to have substantial inadvertent damage to area TE in the right hemisphere, and some hippocampal atrophy in the right hemisphere as well. During surgery, this monkey was noted to have tough, yellow tissue in the right temporal lobe, involving the perirhinal cortex, suggesting that the hippocampal atrophy, like the abnormal tissue, predated the surgery. Hippocampal damage was not evident in any other case.

\section{Cognitive testing}

Experiment 1

Five of the six PRh monkeys reached the criterion for matchingto-sample tests. PRh-D performed consistently $<70 \%$ of the time over 70 sessions of remedial training with only one distracter. $\mathrm{He}$ was then advanced to delay testing despite not having met the criterion.

The first 5 sessions of delay testing were excluded from analysis to ensure that the remaining 10 sessions represented asymptotic performance across delays. The average time elapsed between sample offset and completion of the test were $2.96 \pm$ $0.68,5.62 \pm 0.85,7.88 \pm 1.18,12.80 \pm 2.65,21.19 \pm 2.32$, and $37.93 \pm 2.83 \mathrm{~s}$ (mean $\pm \mathrm{SE}$ ) on each delay level, respectively. Because monkey PRh-D behaved differently than other monkeys in the operated group, we analyzed the results both with and 
Intended
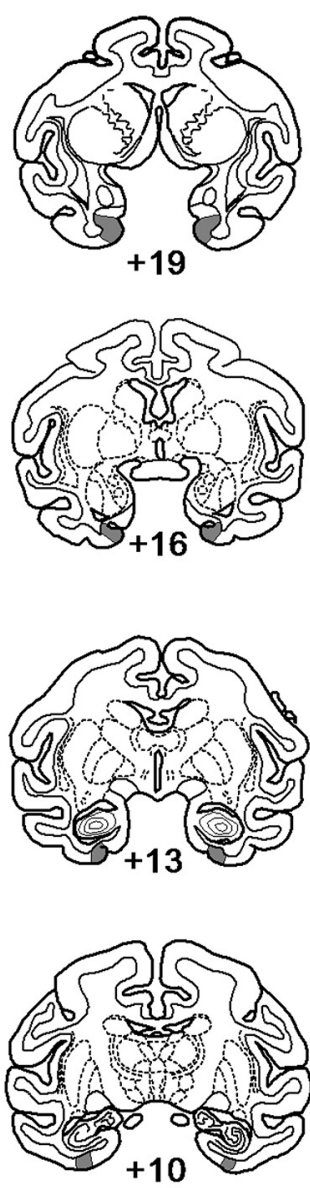

MRI
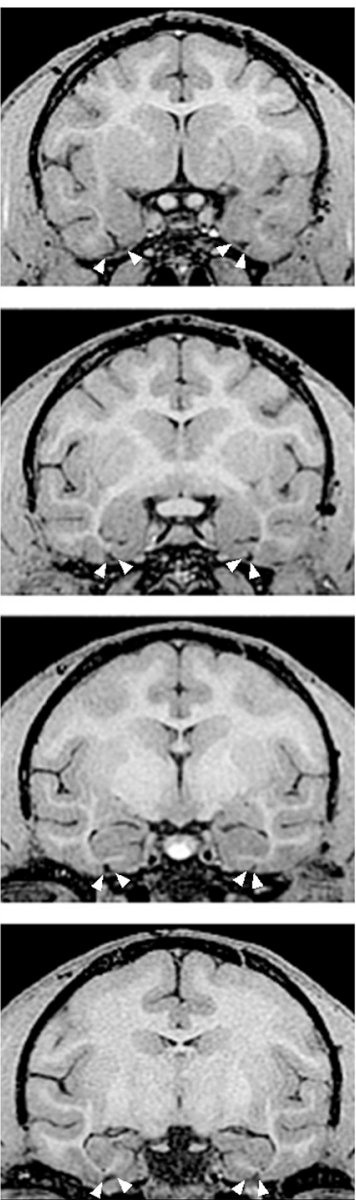

Case PRh-E
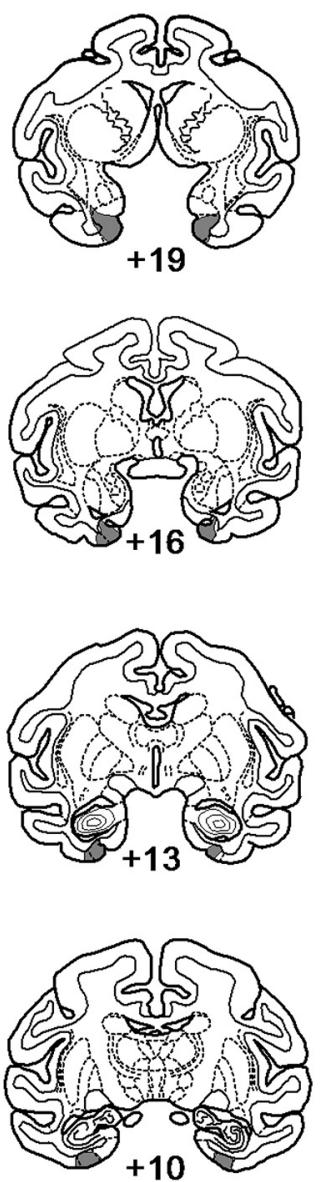

Figure 2. Location and extent of the perirhinal cortex lesion in PRh-E. The intended lesion of perirhinal cortex (shaded region) is shown on coronal sections from a standard rhesus monkey brain (left column). Postoperative MR images from matching levels (middle column) and plots of the lesion (shaded region) onto sections (right column) show the extent of the lesion in PRh-E. Numerals indicate distance in millimeters from the interaural plane. White arrows in the MR images show the boundaries of the lesion.

without his data included (Fig. 3). Repeated-measures ANOVA showed significant interactions between group and delay, as well as the main effects of both these factors, indicating that with longer delays, performance of the PRh monkeys deteriorated faster than that of control monkeys (with PRh-D included: group, $F_{(1,8)}=31.96, p<0.001$; delay, $F_{(5,40)}=37.90, p<0.001$; group $\times$ delay, $F_{(5,40)}=11.24, p<0.001$; with PRh-D excluded: group, $F_{(1,7)}=31.09, p<0.01$; delay, $F_{(5,35)}=39.74$, $p<0.001$; group $\times$ delay, $\left.F_{(5,35)}=11.38, p<0.001\right)$.

\section{Experiment 2}

The critical measures for the PDP analyses come from the probe trials (Table 2). The interaction between group and process was significant both with and without PRh-D (Fig. 4) (repeatedmeasures ANOVA, with PRh-D included: group, $F_{(1,8)}=4.09, p=$ 0.08 ; process, $F_{(1,8)}=0.76, p=0.41$; group $\times$ process, $F_{(1,8)}=9.36$, $p<0.05$; with PRh-D excluded: group, $F_{(1,7)}=3.59, p=0.10$; process, $F_{(1,7)}=0.59, p=0.47$; group $\times$ process, $F_{(1,7)}=7.48, p<$ $0.05)$. Post hoc $t$ tests showed that the two groups of monkeys did not differ on the habit measure (with PRh-D included, $t_{(8)}=1.30, p=$ 0.23 ; with PRh-D excluded, $t_{(7)}=1.03, p=0.34$ ). In contrast, monkeys in the control group had significantly higher one-trial memory scores (with PRh-D included, $t_{(8)}=2.59$, $p<0.05$; with PRh-D excluded, $t_{(7)}=2.36$, $p=0.05)$.

All monkeys selected the high-frequency image more often than expected by chance $(0.25)$ on empty probes (Control: mean $=$ $0.37, t_{(3)}=5.46, p<0.05 ;$ PRh with PRh-D included: mean $=0.41, t_{(5)}=6.28, p<0.01$; PRh with PRh-D excluded: mean $=0.40$, $\left.t_{(4)}=5.10, p<0.01\right)$, and the two groups did not differ in this tendency (with PRh-D included: $t_{(8)}=1.02, p=0.34$; with PRh-D excluded: $\left.t_{(7)}=0.76, p=0.47\right)$. The probability of selecting the high-frequency image on an empty probe trial correlated significantly with the habit measure (with PRh-D included: $r=0.85, p<0.01$; with PRh-D excluded: $r=0.84, p<0.01$ ).

\section{Experiment 3}

Training with extreme bias of $100 \%$ should increase the habit measure but leave the one-trial memory score unaffected. However, one control monkey showed an abrupt and considerable decrease in the one-trial memory score, dropping to almost 0 . To ensure that we collected a representative memory score from this monkey, it was retrained using identical procedures for another 15 sessions, and probe testing was repeated. To match this additional training, the two $\mathrm{PRh}$ monkeys with the lowest one-trial memory scores were also trained and tested again. For all three subjects, we used the higher of the two scores from the two runs in the final analyses.

Compared with the $70 \%$ bias condition in Experiment 2, the $100 \%$ bias condition yielded habit scores that were modestly but significantly higher in the PRh group (Table 3) (paired $t$ tests, with PRh-D included: mean before $=0.38$, mean after $=0.44, t_{5}=$ $8.04, p<0.001$; with PRh-D excluded: mean before $=0.37$, mean after $\left.=0.44, t_{(4)}=8.89, p<0.01\right)$ but not in the control group (paired $t$ test: mean before $=0.34$, mean after $=0.37, t_{(3)}=1.16$, $p=0.33)$. One-trial memory scores did not change significantly in either group (paired $t$ tests, control: mean before $=0.46$, mean after $=0.48, t_{(3)}=0.13, p=0.91$; PRh with PRh-D included: mean before $=0.19$, after $=0.21, t_{(5)}=0.50, p=0.64$; PRh with PRh-D excluded: mean before $=0.19$, after $=0.23, t_{(4)}=1.28$, $p=0.27$ ).

Replicating the results of Experiment 2, there was no main effect of group or process, but the interaction of these two factors was significant (Fig. 5) (repeated-measures ANOVA, with PRh-D included: group, $F_{(1,8)}=3.54, p=0.10$; process, $F_{(1,8)}=1.59, p=$ 0.24 ; group $\times$ process, $F_{(1,8)}=7.83, p<0.05$; with PRh-D excluded: group, $F_{(1,7)}=2.39, p=0.17$; process, $F_{(1,7)}=0.93$, $p=0.37$; group $\times$ process, $\left.F_{(1,7)}=5.83, p<0.05\right)$. Post hoc $t$ tests showed that control monkeys scored higher than PRh monkeys on the one-trial memory measure when PRh-D was included $\left(t_{(8)}=2.46, p<0.05\right)$, but the difference in one-trial memory scores in this post hoc test when PRh-D was excluded did not quite 
Table 1. Damage to area TG, entorhinal cortex, perirhinal cortex, and area TE in six monkeys with perirhinal cortex lesions

\begin{tabular}{|c|c|c|c|c|c|c|c|c|c|c|c|c|}
\hline \multirow[b]{2}{*}{ Monkey } & \multicolumn{4}{|c|}{ Left hemisphere } & \multicolumn{4}{|c|}{ Right hemisphere } & \multicolumn{4}{|c|}{ Average damage } \\
\hline & PRh & ERh & TE & TG & PRh & ERh & TE & TG & PRh & ERh & TE & TG \\
\hline PRh-A & 90.5 & 1.9 & 0.1 & 8.4 & 92.2 & 18.1 & 0.4 & 3.5 & 91.3 & 10.0 & 0.2 & 6.0 \\
\hline PRh-B & 95.8 & 0.0 & 0.4 & 9.6 & 72.1 & 0.0 & 0.0 & 13.6 & 84.0 & 0.0 & 0.2 & 11.6 \\
\hline PRh-C & 86.4 & 4.0 & 3.8 & 7.6 & 88.8 & 0.0 & 25.4 & 0.0 & 87.6 & 2.0 & 14.6 & 3.8 \\
\hline PRh-D & 92.1 & 0.5 & 1.6 & 13.5 & 89.0 & 0.0 & 1.9 & 21.4 & 90.5 & 0.2 & 1.8 & 17.5 \\
\hline PRh-E & 90.9 & 1.6 & 1.5 & 21.3 & 87.4 & 0.0 & 0.3 & 13.3 & 89.1 & 0.8 & 0.9 & 17.3 \\
\hline PRh-F & 86.9 & 6.5 & 5.3 & 18.1 & 98.0 & 2.8 & 2.9 & 7.4 & 92.5 & 4.6 & 4.1 & 12.8 \\
\hline Mean & 90.4 & 2.4 & 2.1 & 13.1 & 87.9 & 3.5 & 5.2 & 9.9 & 89.2 & 3.0 & 3.7 & 11.5 \\
\hline
\end{tabular}

Values are given as percentages. ERh, Entorhinal cortex.

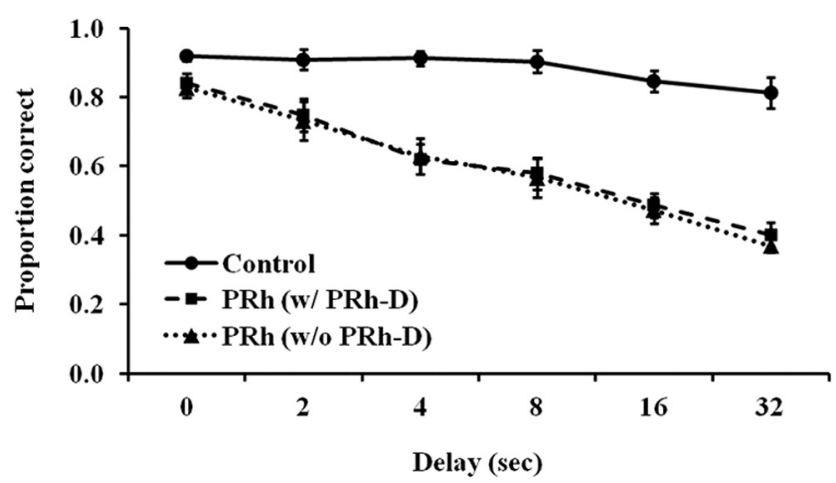

Figure 3. Average matching-to-sample performance with different delays in control monkeys ( $n=4$; solid line) and monkeys from which perirhinal cortex was removed bilaterally, with all PRh monkeys ( $n=6$; dashed line) and with PRh-D excluded ( $n=5$; dotted line). Error bars indicate SEs.

Table 2. Probability of choosing the high-frequency image on congruent and incongruent probes and derived PDP scores in Experiment 2 (70\% bias)

\begin{tabular}{cllll}
\hline & Congruent & Incongruent & One-trial & Habit \\
\hline Con-A & 0.58 & 0.21 & 0.37 & 0.33 \\
Con-B & 0.63 & 0.24 & 0.39 & 0.39 \\
Con-C & 0.78 & 0.10 & 0.68 & 0.31 \\
Con-D & 0.60 & 0.19 & 0.41 & 0.32 \\
Mean & 0.65 & 0.19 & 0.46 & 0.34 \\
PRh-A & 0.48 & 0.35 & 0.13 & 0.40 \\
PRh-B & 0.42 & 0.28 & 0.14 & 0.33 \\
PRh-C & 0.74 & 0.19 & 0.55 & 0.42 \\
PRh-D & 0.52 & 0.33 & 0.19 & 0.41 \\
PRh-E & 0.33 & 0.27 & 0.06 & 0.29 \\
PRh-F & 0.48 & 0.39 & 0.09 & 0.43 \\
Mean & 0.50 & 0.30 & 0.19 & 0.38 \\
\hline
\end{tabular}

Note that in the congruent condition, values are equivalent to the percentage correct, while in the incongruent condition it reflects the probability of erring by selecting the high-frequency image (and not one of the other low-frequency images). Con, Control.

reach significance $\left(t_{(7)}=2.10, p=0.07\right)$. There was no significant difference in habit scores with or without PRh-D (with PRh-D included: $t_{(8)}=1.99, p=0.08$; with PRh-D excluded: $t_{(7)}=1.76$, $p=0.12$ ).

As in Experiment 2, both groups of monkeys were numerically more likely to select the high-frequency image than expected by chance (0.25) on empty probes, although the tendency was not quite significant in the control group (control: mean $=0.43, t_{(3)}=3.06, p=0.06$; PRh with PRh-D included: mean $=0.44, t_{(5)}=7.17, p<0.01 ; \mathrm{PRh}$ with PRh-D excluded: mean $\left.=0.44, t_{(4)}=5.92, p<0.01\right)$. The two groups did not differ in this tendency (with PRh-D included: $t_{(8)}=$ 0.29, $p=0.78$; with PRh-D excluded: $t_{(7)}=0.29, p=0.78$ ). The probability of selecting the high-frequency image on an

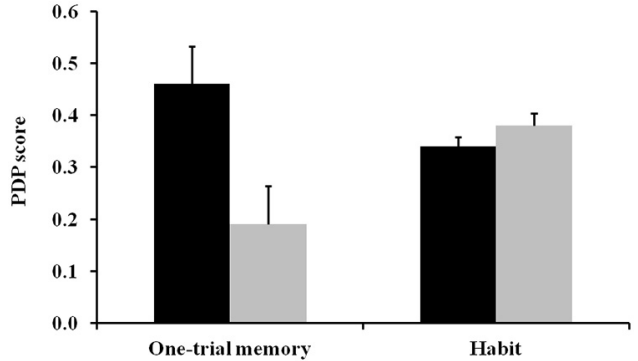

Figure 4. The contributions of one-trial memory and habit to matching-to-sample performance in control monkeys (black bars) and monkeys from which perirhinal cortex was removed bilaterally, including PRh-D (gray bars). Habits were produced over 25 sessions in which highfrequency images were rewarded on 70\% of trials in Experiment 2. Error bars indicate SEs. See text for details of the process dissociation procedure analysis used to generate the values.

Table 3. Probability of choosing the high-frequency image on congruent and incongruent probes and derived PDP scores in Experiment 3 ( $100 \%$ bias)

\begin{tabular}{cllll}
\hline & Congruent & Incongruent & One-trial & Habit \\
\hline Con-A & 0.63 & 0.27 & 0.36 & 0.42 \\
Con-B & 0.53 & 0.30 & 0.23 & 0.39 \\
Con-C & 0.79 & 0.08 & 0.71 & 0.28 \\
Con-D & 0.76 & 0.16 & 0.60 & 0.40 \\
Mean & 0.68 & 0.20 & 0.48 & 0.37 \\
PRh-A & 0.56 & 0.40 & 0.16 & 0.48 \\
PRh-B & 0.51 & 0.32 & 0.19 & 0.40 \\
PRh-C & 0.71 & 0.25 & 0.46 & 0.46 \\
PRh-D & 0.50 & 0.41 & 0.09 & 0.45 \\
PRh-E & 0.43 & 0.33 & 0.10 & 0.37 \\
PRh-F & 0.62 & 0.38 & 0.24 & 0.50 \\
Mean & 0.56 & 0.35 & 0.21 & 0.44 \\
\hline
\end{tabular}

Note that in the congruent condition, values are equivalent to the percentage correct, while in the incongruent condition it reflects the probability of erring by selecting the high-frequency image (and not one of the other low-frequency images). Con, Control.

empty probe again correlated significantly with the habit measure (with PRh-D included: $r=0.81, p<0.01$; with PRh-D excluded: $r=0.83, p<0.01)$.

\section{Discussion}

PDP resulted in a quantitative dissociation of one-trial memory and habit within a single cognitive test in monkeys. This dissociation is particularly informative because the two types of memory were measured in the identical task context, using identical visual stimuli, in tests with the same subjects, precisely controlling for a host of factors that might cause differences in performance across separate memory tests. This establishes a clear correspondence in monkeys to results from human PDP tests in which one-trial memory has been dissociated from habits acquired over multiple trials (Jacoby, 1991; Hay and Jacoby, 1996). 


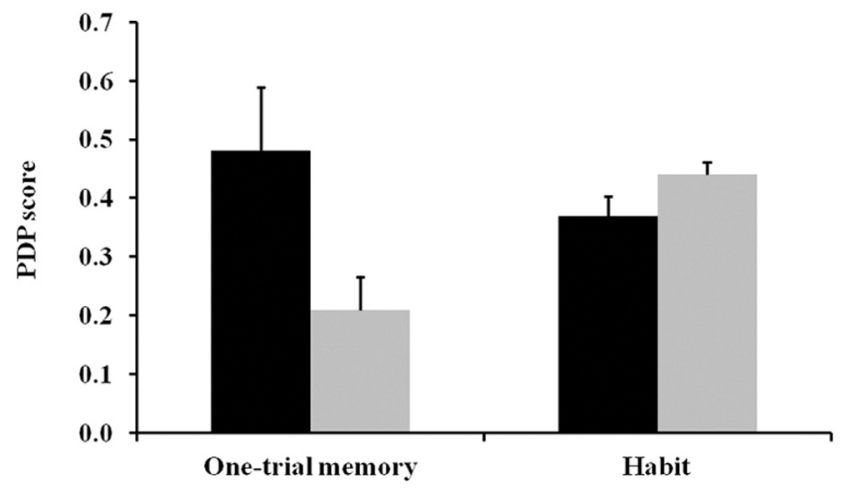

Figure 5. The contribution of one-trial memory and habit to matching-to-sample performance in control monkeys (black bars) and monkeys from which perirhinal cortex was removed bilaterally, including PRh-D (gray bars). Habits were further developed over 15 sessions in which high-frequency images were rewarded on $100 \%$ of trials in Experiment 3. Error bars indicate SEs. See text for details of the process dissociation procedure analysis used to generate the values.

In this first use of PDP with monkeys, it was important to validate our measures. Experiment 1 revealed that the PRh group rapidly forgot sample images, consistent with the lower one-trial memory scores resulting from PDP in Experiments 2 and 3. Performance on empty probe trials, which depends on a history of repeated reinforcement as is the case in object discrimination learning, correlated with the habit scores. A future study of whether damage to another region, such as tail of the caudate nucleus (Fernandez-Ruiz et al., 2001), would selectively impair habit scores, would test for a double dissociation and would further evaluate the suitability of PDP in monkeys.

PDP generated an especially clear distinction regarding the role of perirhinal cortex in memory performance. Perirhinal cortex in monkeys receives strong input from the ventral visual pathway and projects heavily to the hippocampus via the entorhinal cortex (Suzuki, 1996). This anatomical position is consistent with both high-level perceptual functions and memory function (Baxter, 2009; Suzuki, 2010). It is established that perirhinal cortex is critical for performance in memory tests (Meunier et al., 1993; Buffalo et al., 1999) and also contributes to discrimination learning under some conditions (Buckley and Gaffan, 1997, 1998; Bussey et al., 2002; Hampton and Murray, 2002). We found that one-trial memory was impaired by PRh lesions, but habit formation was intact, even though these capacities required equivalent perceptual function to discriminate test images. This dissociation reinforces the importance of mnemonic functions of perirhinal cortex. Other reports indicate a role for perirhinal cortex in the acquisition of some visual discriminations, which presumably requires the development of habits (Buckley and Gaffan, 1997, 1998; Bussey et al., 2002; Hampton and Murray, 2002), and for perirhinal cortex together with entorhinal cortex in the retention of preoperatively acquired visual discriminations (Gaffan and Murray, 1992; Thornton et al., 1997). We did not measure the course of habit acquisition in this study and cannot determine whether perirhinal cortex removal impacted the acquisition of the habits. The dissociation we report here does clearly show that deficits in onetrial memory following perirhinal cortex removal occur with a large set of visual stimuli that monkeys can discriminate without impairment in the execution of postoperatively acquired visual habits.
Because it is unlikely that cognitive tests measure just a single cognitive process, application of PDP in other paradigms might help resolve some apparent contradictions in the literature. For example, concurrent discrimination has been viewed as habit learning because monkeys with medial temporal lobe lesions performed normally in this task (Malamut et al., 1984). However, given the same task, amnesic patients with medial temporal lobe damage showed absence of learning (Squire et al., 1988; Hood et al., 1999). Studies have suggested that two fundamentally different strategies may be used to solve a concurrent discrimination task (Buffalo et al., 1998; Hood et al., 1999). One is to explicitly remember the specific features of the rewarded item in each pair, an ability that is thought to depend on certain medial temporal lobe structures. Human patients may adopt this approach (Squire and ZolaMorgan, 1991; Hood et al., 1999). The other involves gradual acquisition of dispositions to choose the rewarded object in each pair, a process that can occur independently of the medial temporal lobe and may be the method adopted by monkeys (Malamut et al., 1984; Squire and Zola-Morgan, 1991). Furthermore, even with the same task, parametric differences may cloud comparisons between studies. For instance, the frequency of each stimulus pair in a concurrent discrimination task may change the relative contribution of one-trial memory and habit. If each discrimination is seen more than once in a session (Squire et al., 1988), one-trial memory may contribute to performance, whereas this is unlikely in experiments where each pair is only used once per day (Malamut et al., 1984). PDP paradigms make it possible to measure the relative contributions of both of these types of memory to learning concurrent discriminations, across variations in studies.

Differences in cognitive strategy may be reflected in PDP scores. One control monkey showed a memory score of almost 0 in the first round of testing in Experiment 3, suggesting that monkeys may use one-trial memory when the identity of the sample matters, as in normal matching-to-sample or in the $70 \%$ bias condition of Experiment 2, but may abandon one-trial memory when it is irrelevant, as in the $100 \%$ bias condition. The habit scores of PRh monkeys, but not those of control monkeys, increased modestly but significantly after extremely biased training in Experiment 3. This may suggest that the impairment in one-trial memory, combined with sparing of habit acquisition, disposes monkeys lacking perirhinal cortex to express habits more readily than do control monkeys. Whether this result represents a controlled, strategic process, or rather the absence of an overshadowing effect of one-trial memory on the expression of habits, is a question that deserves to be addressed in further research. It is noteworthy that habit scores never approached the maximum of 1.0, even in Experiment 3 in which monkeys could have solved $100 \%$ of training trials correctly using habit. Instead, one-trial memory continued to contribute to performance. This suggests that monkeys approach the task as a one-trial memory task and habits emerge only when memory fails. It also suggests that habit is not "easier" for monkeys than is one-trial memory, so difficulty would not account for the selective impairment of one-trial memory.

PDP has been used to dissociate other memory types, for example, recollection and familiarity in humans (McCabe et al., 2011). Validation of PDP in monkeys may create new opportunities for the study of other memory systems in monkeys, but the use of PDP is limited by several factors. First, all uses of PDP depend on a contrast between two processes, one under cognitive control and one not. Second, PDP does not 
work when performance is determined by more than two cognitive processes acting concurrently. Third, PDP depends on the assumption that the cognitive processes being measured are independent. Some authors have argued that violations of this assumption may lead to artificial dissociations (Joordens and Merikle, 1993; Curran and Hintzman, 1995). Jacoby et al. (1997) have countered that correlations between processes only produce negligible biases that are insufficient to generate artificial dissociations. On their account, such "paradoxical dissociations" instead result from floor effects or other violations of the test conditions that can be avoided.

Establishing correspondences between human and nonhuman memory is a major challenge in the development of valid animal models of human memory, and distinguishing between controlled and automatic memory processes in nonverbal animals is difficult. In humans, declarative or explicit memories are consciously accessible, reflecting the ability of subjects to monitor and control the use of these memories in the expression of behavior. Nondeclarative or implicit memories can be inferred from their influence on behavior, but cannot be brought to conscious awareness (Tulving and Schacter, 1990; Squire et al., 1993; Cohen and Eichenbaum, 1994). This is reflected in findings that the influence of these memories on behavior proceeds automatically, without cognitive control (Jacoby, 1991; Jacoby et al., 1993). Declarative and nondeclarative memories are often distinguished in humans on the basis of verbal commentary, for example, "I remembered" versus "I guessed." While some results suggest that monkeys possess declarative memories, in the sense that they can monitor the presence of these memories (Smith et al., 1998; Hampton, 2001; Kornell et al., 2007), interpretation of such findings is complicated and converging evidence will be required (Hampton, 2009; Smith, 2009; Murray and Wise, 2010). Because PDP in humans does not depend on verbal commentary that nonhumans cannot produce, it provides an approach to establishing correspondences between human and nonhuman automatic and controlled memory systems. Future research should address whether the one-trial and habit memory systems identified here with PDP differ in accessibility to cognitive monitoring in monkeys.

PDP is a valuable behavioral technique in humans that dissociates automatic and controlled memory systems (Jacoby, 1991; Jacoby et al., 1993). Here we showed that PDP also provides a powerful experimental tool for studies of nonhuman species that cannot verbally describe their experience of memory as do humans. Dissociations between memory systems in nonhuman primates are critical for understanding the neurobiological mechanisms supporting cognition in humans. Understanding the neurobiology of explicit and implicit cognition in nonhumans is particularly challenging, and PDP provides a new approach to the problem.

\section{References}

Bachevalier J (1990) Ontogenetic development of habit and memory formation in primates. Ann N Y Acad Sci 608:457-477.

Bachevalier J, Mishkin M (1994) Effects of selective neonatal temporal lobe lesions on visual recognition memory in rhesus monkeys. J Neurosci 14:2128-2139.

Bachevalier J, Beauregard M, Alvarado MC (1999) Long-term effects of neonatal damage to the hippocampal formation and amygdaloid complex on object discrimination and object recognition in rhesus monkeys (Macaca mulatta). Behav Neurosci 113:1127-1151.

Baxter MG (2009) Involvement of medial temporal lobe structures in memory and perception. Neuron 61:667-677.

Buckley MJ, Gaffan D (1997) Impairment of visual object-discrimination learning after perirhinal cortex ablation. Behav Neurosci 111:467-475.
Buckley MJ, Gaffan D (1998) Perirhinal cortex ablation impairs visual object identification. J Neurosci 18:2268-2275.

Buffalo EA, Stefanacci L, Squire LR, Zola SM (1998) A reexamination of the concurrent discrimination learning task: the importance of anterior inferotemporal cortex, area TE. Behav Neurosci 112:3-14.

Buffalo EA, Ramus SJ, Clark RE, Teng E, Squire LR, Zola SM (1999) Dissociation between the effects of damage to perirhinal cortex and area TE. Learn Mem 6:572-599.

Bussey TJ, Saksida LM, Murray EA (2002) Perirhinal cortex resolves feature ambiguity in complex visual discriminations. Eur J Neurosci 15:365-374.

Cohen NH, Eichenbaum H (1994) Memory, amnesia, and the hippocampal system. Cambridge, MA: MIT.

Curran T, Hintzman DL (1995) Violations of the independence assumption in process dissociation. J Exp Psychol Learn Mem Cogn 21:531-547.

Fernandez-Ruiz J, Wang J, Aigner TG, Mishkin M (2001) Visual habit formation in monkeys with neurotoxic lesions of the ventrocaudal neostriatum. Proc Natl Acad Sci U S A 98:4196-4201.

Gaffan D, Murray EA (1992) Monkeys (Macaca fascicularis) with rhinal cortex ablations succeed in object discrimination learning despite 24-h intertrial intervals and fail at matching to sample despite double sample presentations. Behav Neurosci 106:30-38.

Hampton RR (2001) Rhesus monkeys know when they remember. Proc Natl Acad Sci U S A 98:5359-5362.

Hampton RR (2009) Multiple demonstrations of metacognition in nonhumans: converging evidence or multiple mechanisms? Comp Cogn Behav Rev 4:17-28.

Hampton RR, Murray EA (2002) Learning of discriminations is impaired, but generalization to altered views is intact, in monkeys (Macaca mulatta) with perirhinal cortex removal. Behav Neurosci 116:363-377.

Harlow HF (1949) The formation of learning sets. Psychol Rev 56:51-65.

Hay JF, Jacoby LL (1996) Separating habit and recollection: memory slips, process dissociations, and probability matching. J Exp Psychol Learn Mem Cogn 22:1323-1335.

Henke K (2010) A model for memory systems based on processing modes rather than consciousness. Nat Rev Neurosci 11:523-532.

Hood KL, Postle BR, Corkin S (1999) An evaluation of the concurrent discrimination task as a measure of habit learning: performance of amnesic subjects. Neuropsychologia 37:1375-1386.

Jacoby LL (1991) A process dissociation framework-separating automatic from intentional uses of memory. J Mem Lang 30:513-541.

Jacoby LL, Toth JP, Yonelinas AP (1993) Separating conscious and unconscious influences of memory: measuring recollection. J Exp Psychol Gen 122:139-154

Jacoby LL, Begg IM, Toth JP (1997) In defense of functional independence: violations of assumptions underlying the process-dissociation procedure? J Exp Psychol Learn Mem Cogn 23:484-495.

Joordens S, Merikle PM (1993) Independence or redundancy? Two models of conscious and unconscious influences. J Exp Psychol Gen 122:462-467.

Kelley CM, Jacoby LL (1998) Subjective reports and process dissociation: fluency, knowing, and feeling. Acta Psychol 98:127-140.

Kelley CM, Jacoby LL (2000) Recollection and familiarity. In: The Oxford handbook of memory (Tulving E, Craik FIM, eds), pp 215-228. New York: Oxford UP.

Kirk RE (1982) Experimental design, Ed2. Belmont, CA: Wadsworth.

Kornell N, Son LK, Terrace HS (2007) Transfer of metacognitive skills and hint seeking in monkeys. Psychol Sci 18:64-71.

Liu Z, Murray EA, Richmond BJ (2000) Learning motivational significance of visual cues for reward schedules requires rhinal cortex. Nat Neurosci 3:1307-1315.

Malamut BL, Saunders RC, Mishkin M (1984) Monkeys with combined amygdalo-hippocampal lesions succeed in object discriminationlearning despite 24-hour intertribal intervals. Behav Neurosci 98:759-769.

Málková L, Lex CK, Mishkin M, Saunders RC (2001) MRI-based evaluation of locus and extent of neurotoxic lesions in monkeys. Hippocampus 11:361-370

McCabe DP, Roediger HL 3rd, Karpicke JD (2011) Automatic processing influences free recall: converging evidence from the process dissociation procedure and remember-know judgments. Mem Cognit 39:389-402.

Meunier M, Bachevalier J, Mishkin M, Murray EA (1993) Effects on visual 
recognition of combined and separate ablations of the entorhinal and perirhinal cortex in rhesus monkeys. J Neurosci 13:5418-5432.

Mishkin M, Malamut B, Bachevalier J (1984) Memories and habits: two neural systems. In: Neurobiology of learning and memory (Lynch G, McGaugh JL, Weinberger NM, eds), pp 65-77. New York: Guilford.

Murray EA, Wise SP (2010) What, if anything, can monkeys tell us about human amnesia when they can't say anything at all? Neuropsychologia 48:2385-2405.

Reingold EM, Goshen-Gottstein YG (1996) Separating consciously controlled and automatic influences in memory for new associations. J Exp Psychol Learn Mem Cogn 22:397-406.

Schrier AM (1966) Transfer by macaque monkeys between learning set and repeated-reversal tasks. Percept Mot Skills 23:787-792.

Smith JD (2009) The study of animal metacognition. Trends Cogn Sci 13:389-396.

Smith JD, Shields WE, Allendoerfer KR, Washburn DA (1998) Memory monitoring by animals and humans. J Exp Psychol Gen 127:227-250.

Squire LR, Zola-Morgan S (1991) The medial temporal lobe memory system. Science 253:1380-1386.

Squire LR, Zola-Morgan S, Chen KS (1988) Human amnesia and animal models of amnesia: performance of amnesic patients on tests designed for the monkey. Behav Neurosci 102:210-221.

Squire LR, Knowlton B, Musen G (1993) The structure and organization of memory. Annu Rev Psychol 44:453-495.

Suzuki WA (1996) Neuroanatomy of the monkey entorhinal, perirhinal and parahippocampal cortices: organization of cortical inputs and interconnections with amygdala and striatum. Semin Neurosci 8: $3-12$.

Suzuki WA (2010) Untangling memory from perception in the medial temporal lobe. Trends Cogn Sci 14:195-200.

Thornton JA, Rothblat LA, Murray EA (1997) Rhinal cortex removal produces amnesia for preoperatively learned discrimination problems but fails to disrupt postoperative acquisition and retention in rhesus monkeys. J Neurosci 17:8536-8549.

Toth JP, Reingold EM, Jacoby LL (1994) Towards a redefinition of implicit memory: process dissociations following elaborative processing and selfgeneration. J Exp Psychol Learn Mem Cogn 20:290-303.

Tulving E, Schacter DL (1990) Priming and human memory systems. Science 247:301-306. 